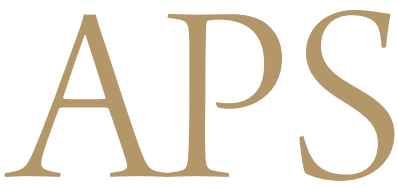

Archives of Plastic Surgery

\title{
Clinical Experiences with the Scapular Fascial Free Flap
}

\author{
Il Ho Park, Chul Hoon Chung, Yong Joon Chang, Jae Hyun Kim \\ Department of Plastic and Reconstructive Surgery, Hallym University College of Medicine, Seoul, Korea
}

Background The goal of reconstruction is to provide coverage of exposed vital structures with well-vascularized tissue for optimal restoration of form and function. Here, we present our clinical experience with the use of the scapular fascial free flap to correct facial asymmetry and to reconstruct soft tissue defects of the extremities.

Methods We used a scapular fascial free flap in 12 cases for soft tissue coverage of the extremities or facial soft tissue augmentation.

Results The flaps ranged in size from $3 \times 12$ to $13 \times 23 \mathrm{~cm}$. No cases of total loss of the flap occurred. Partial loss of the flap occurred in 1 patient, who was treated with a turnover flap using the adjacent scapular fascial flap and a skin graft. Partial loss of the skin graft occurred in 4 patients due to infection or hematoma beneath the graft, and these patients underwent another skin graft. Four cases of seroma at the donor site occurred, and these cases were treated with conservative management or capsulectomy and quilting sutures.

Conclusions The scapular fascial free flap has many advantages, including a durable surface for restoration of form and contours, a large size with a constant pedicle, adequate surface for tendon gliding, and minimal donor-site scarring. We conclude that despite the occurrence of a small number of complications, the scapular fascial free flap should be considered to be a viable option for soft tissue coverage of the extremities and facial soft tissue augmentation.

Keywords Free tissue flaps / Facial asymmetry / Soft tissue injury

\author{
Correspondence: Chul Hoon Chung \\ Department of Plastic and \\ Reconstructive Surgery, Hallym \\ University College of Medicine, 150 \\ Seongan-ro, Gangdong-gu, Seoul \\ 05355, Korea \\ Tel: +82-2-2224-2246 \\ Fax: +82-2-489-0010 \\ E-mail:c21ps@hanmail.net
}

\begin{abstract}
This article was presented as a freestanding paper at the 72nd Congress of the Korean Society of Plastic and Reconstructive Surgeons on November 7, 2014 in Seoul, Korea.
\end{abstract}

No potential conflict of interest relevant to this article was reported.

\section{INTRODUCTION}

With the emergence of more detailed knowledge of human anatomy and gradual advancements in microsurgical technology, reconstructive surgeons have begun to consider a range of characteristics of free flaps, such as flap composition and reliability, functional and aesthetic results of the recipient site, and donor-site morbidity. Surgeons have sought to identify the free flaps most suitable for specific recipient sites.

With regard to the fascial free flap, in 1980, Smith [1] used the temporoparietal fascia to reconstruct soft tissue defects of the lower extremities, and reported that this fascia was advantageous due to being thin but durable However, it is difficult to use the temporoparietal fascia to augment facial soft tissue or to resurface large soft tissue defects due to its thinness and restricted size. In 1987, Kim et al. [2] demonstrated that the dorsal thoracic fascia, included within the widely used scapular/parascapular flap, may be used as a fascial flap with the circumflex scapular vessels; this type of fascial flap has been referred to as the dorsal thoracic fascial flap or the scapular fascial flap [3-6]. The 
scapular fascial flap has several advantages over the temporoparietal fascial flap; for instance, the scapular fascia can yield a larger and thicker flap that can be used to reconstruct large soft tissue defects of the extremities or to augment facial soft tissue. However, despite these merits, the scapular fascial flap has not been commonly used in Korea. Globally, except for some reports describing the use of the scapular fascial flap for the reconstruction of large soft tissue defects of the extremities or facial soft tissue augmentation, no studies have comprehensively analyzed its various clinical applications.

In this study, we analyzed the outcomes of patients treated with a scapular fascial free flap with the aim of reconsidering the applicability of this flap.

\section{METHODS}

We performed a retrospective study analyzing the medical records of 12 patients treated with a scapular fascial free flap by a single surgeon from 1994 to 2013.

Ten patients were male and 2 were female, with an average age of 34.1 years (range, 11-57 years). All patients were free of underlying diseases such as hypertension, diabetes mellitus, and vascular disease. A scapular fascial free flap was used for facial soft tissue augmentation in 3 cases and for the reconstruction of soft tissue defects of the extremities in 9 cases. The causes of the soft tissue defects of the extremities included electrical burns $(\mathrm{n}=6)$, an injury from a hot roller $(\mathrm{n}=1)$, an injury from a conveyer belt $(n=1)$, and postburn scar contracture $(n=1)$. Facial soft tissue augmentation was performed in patients with mucoepidermoid carcinoma of the right parotid gland, squamous cell carcinoma of the right maxillary sinus, and facial asymmetry after treatment for an abscess involving the right mandibular area. The recipient sites for the scapular fascial free flap were the dor- sum of the foot $(n=5)$, ankle $(n=2)$, forearm $(n=1)$, hand $(n=1)$, and face $(\mathrm{n}=3)$ (Table 1$)$.

The thickness of the scapular fascia was determined using sonography prior to surgery, and the thickness and size of the scapular fascial flap were measured during surgery. For postoperative monitoring of the flap, in cases of facial soft tissue augmentation, a small piece of skin was included in the flap as a monitoring flap and removed 14 days after surgery. For reconstruction of the extremities, an opening was made in the wound dressing for continuous postoperative monitoring. In addition, we examined the postoperative complications and level of patient satisfaction regarding the functional and aesthetic outcomes of both the recipient and donor sites after an average follow-up of 12 months (range, 2-46 months).

\section{Flap elevation technique}

For scapular fascial flap elevation, the patient was placed in the lateral decubitus position, and the arm was placed in adduction. The scapula and posterior triangle space, comprising the teres major muscle, teres minor muscle, and long head of the triceps muscle, were marked, followed by marking of the transverse and descending branches of the circumflex scapular artery using Doppler signal tracing. The shape of the defect was designed around the vascular pedicle, and an approach to the superficial fascial layer was made via an incision along the long axis of the flap. The superficial fascia was then exposed and undermined between the subcutaneous fat and superficial fascia in order to obtain adequate exposure (Fig. 1).

Flap elevation was performed by first redesigning the fascia according to the defect size, including both descending and transverse branches of the circumflex scapular artery, and then dissecting a fascial flap (including the fat tissue and deep fascia below the superficial fascia) from the medial portion close to the

\section{Table 1. Details of the cases}

\begin{tabular}{|c|c|c|c|c|c|c|}
\hline Patient & Sex/Age (yr) & Cause & Site & $\begin{array}{l}\text { Size of flap } \\
(\mathrm{cm})\end{array}$ & $\begin{array}{l}\text { Thickness of flap } \\
(\mathrm{cm})\end{array}$ & $\begin{array}{c}\text { Follow-up } \\
(\mathrm{mo})\end{array}$ \\
\hline 1 & Male/11 & Conveyor belt & Foot dorsum & $13 \times 9$ & 0.5 & 2 \\
\hline 2 & Male/26 & Electrical burn & Foot dorsum & $15 \times 10$ & 1.0 & 10 \\
\hline 3 & Male/26 & Electrical burn & Foot dorsum & $15 \times 10$ & 1.0 & 46 \\
\hline 4 & Male/30 & Electrical burn & Foot dorsum & $3 \times 12$ & 0.6 & 4 \\
\hline 5 & Male/33 & Electrical burn & Foot dorsum & $11 \times 14$ & 0.8 & 2 \\
\hline 6 & Male/34 & Hot roller & Forearm & $13 \times 23$ & 1.1 & 18 \\
\hline 7 & Male/39 & Infection & Cheek & $7.5 \times 6$ & 0.7 & 4 \\
\hline 8 & Male/42 & Electrical burn & Hand & $10 \times 6$ & 0.9 & 27 \\
\hline 9 & Male/45 & Electrical burn & Ankle & $10 \times 12$ & 1.0 & 5 \\
\hline 10 & Male/57 & Postmaxillectomy deformity & Cheek & $14 \times 10$ & 1.0 & 6 \\
\hline 11 & Female/27 & Postburn scar contracture & Ankle & $11 \times 15$ & 0.9 & 7 \\
\hline 12 & Female/39 & Postparotidectomy deformity & Cheek and neck & $15 \times 9$ & 1.1 & 13 \\
\hline
\end{tabular}




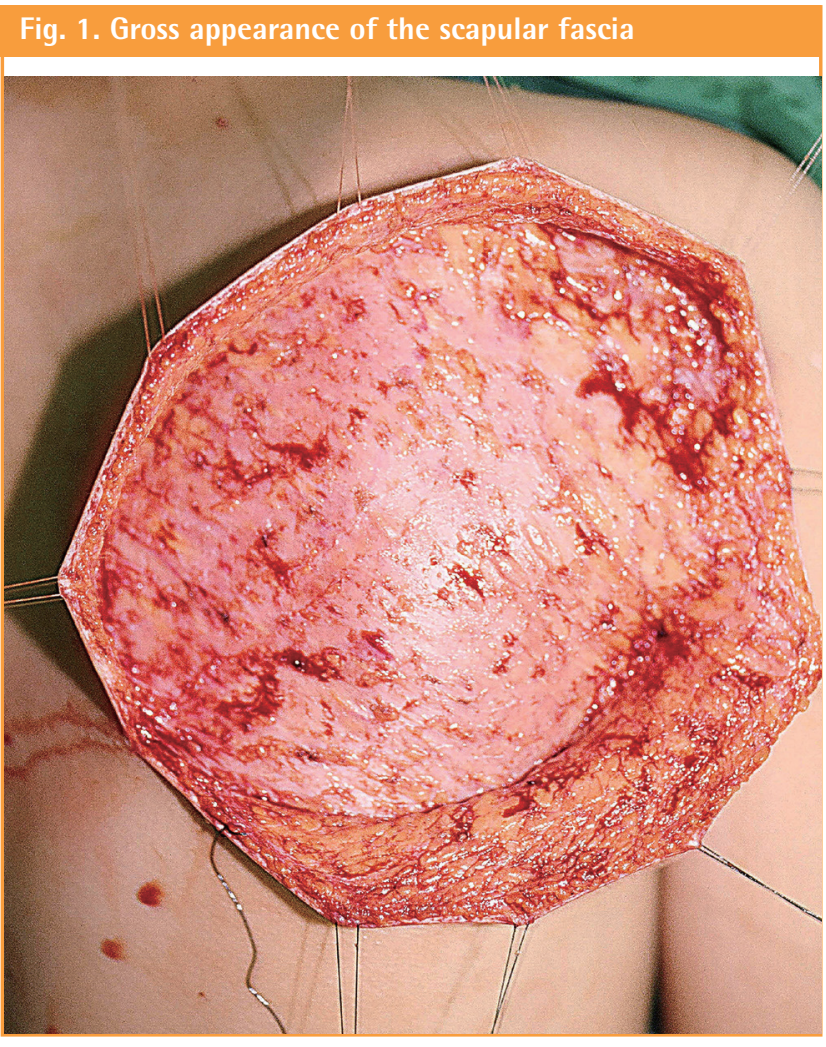

spine to the lateral portion. When performing pedicle dissection in the posterior triangle area following flap elevation, meticulous ligation of the branches leading to the surrounding muscles and scapular bone was performed for thorough bleeding control. If a long pedicle for vascular anastomosis was not necessary, a short pedicle (approximately $5 \mathrm{~cm}$ ) was acquired from the area where the circumflex scapular artery arises from the subscapular artery. If a long pedicle was required for vascular anastomosis, approximately $7-8 \mathrm{~cm}$ was acquired by dissection up to the area where the subscapular artery arises from the axillary artery (Fig. 2).

\section{RESULTS}

The average size and thickness of the scapular fascial free flaps were $130.9 \mathrm{~cm}^{2}$ (range, 36-299 $\mathrm{cm}^{2}$ ) and $0.91 \mathrm{~cm}$ (range, 0.5 $1.1 \mathrm{~cm})$, respectively.

Patients who underwent the reconstruction of soft tissue defects of the extremities had tendons that were damaged in the injury. However, postoperatively, they experienced no specific problems in tendon gliding.

No cases of total loss of the flap were observed. In 1 patient who underwent reconstruction of the left forearm, the distal portion of the flap and skin graft were lost due to infection. In this patient, a turnover flap was performed using an adjuvant scapular fascial flap and another skin graft. In 4 of the 8 patients who underwent the reconstruction of soft tissue defects of the

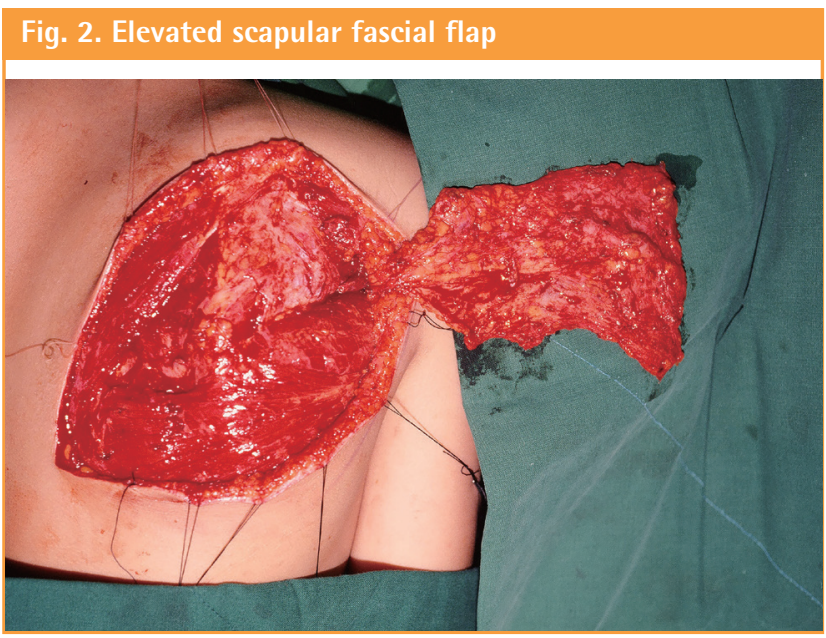

extremities, the initial skin graft used to cover the flap was partially lost, and another skin graft was applied following debridement. In the 3 cases of facial soft tissue augmentation, complications associated with the flap did not occur.

Complications involving the donor site included seroma in 4 cases and hypertrophic scarring in 1 case. Among the 4 patients who developed seroma, 2 were treated with conservative management, while the other 2 were treated with seroma sac remov$\mathrm{al}$ and quilting sutures. Scar revision was performed in the patient who developed a hypertrophic scar.

Other complications included hyperpigmentation of the skin graft covering the flap, and a bulky appearance in patients who underwent dorsal foot reconstruction (due to the flap being thicker than the normal surrounding soft tissue). One patient who underwent forearm reconstruction and 1 patient who underwent foot reconstruction complained of hyperpigmentation. Two of the 5 patients who underwent foot reconstruction complained of bulkiness but did not want corrective surgery.

\section{Case reports}

\section{Case 1}

A 34-year-old man was transferred to our hospital after receiving a skin graft at an outside hospital for a crushing injury of the medial side of the left forearm and wrist by a hot roller. On admission, there was evident necrosis of the exposed flexor digitorum superficialis muscle and tendons and moderate ischemic contracture of the flexor digitorum profundus muscle, which resulted in flexion contracture of 4 digits (excluding the thumb), rendering them almost immobile. This condition was accompanied by a partial defect of the radial artery, median nerve, and superficial radial nerve.

Following debridement of the necrotic tissue, a slide operation was performed on the flexor digitorum profundus muscle, and cable grafts were performed on the damaged area of the median 


\section{Fig. 3. Case 1: partial loss of the flap}

A 34-year-old man with a crushing injury of the left forearm and wrist by a hot roller. (A) Preoperative appearance. (B) Partial loss of the flap and skin graft occurred, and a turnover flap $(5 \times 3 \mathrm{~cm})$ was performed 3 weeks postoperatively. (C) Appearance and function 2 years postoperatively.
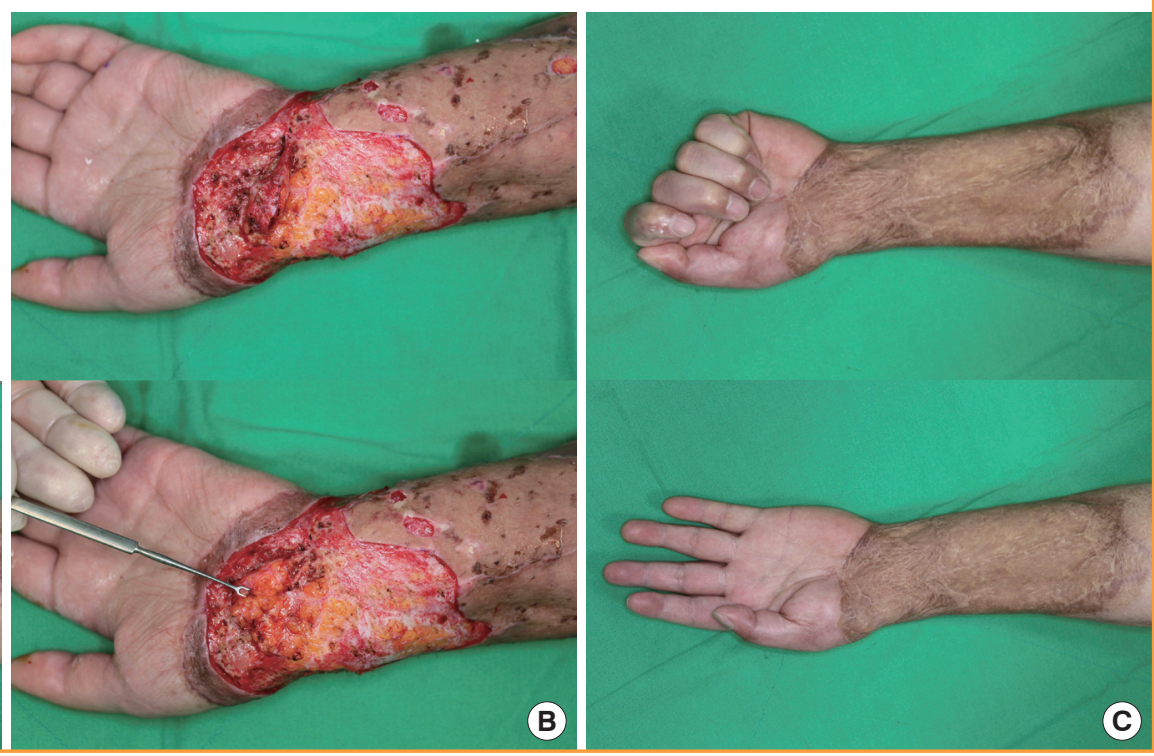

nerve using the sural nerve. The soft tissue defect of the forearm was covered with a $13 \times 23-\mathrm{cm}$ scapular fascial flap elevated from the left back. A split-thickness skin graft (STSG) was then applied on the raw surface of the fascial flap. Recipient vessels included the radial artery, cephalic vein, and 1 vena comitans of the radial artery located on the proximal forearm.

Three weeks after surgery, the distal portion of the flap and skin graft were partially lost due to infection with methicillin-resistant Staphylococcus aureus. To treat this complication, part of the scapular fascial flap near the defect was used to perform a $5 \times 3$ cm turnover flap followed by an STSG (Fig. 3).

\section{Case 2}

A 39-year-old woman presented with a history of total parotidectomy and superior omohyoid neck dissection to treat a mucoepidermoid carcinoma of the right parotid gland 5 years prior. At the time, she underwent simultaneous masseter muscle transfer for the correction of right facial nerve palsy.

Postoperatively, she developed a depressed deformity of the right cheek and upper neck. Soft tissue augmentation was performed with a $15 \times 9-\mathrm{cm}$ scapular fascial free flap that included a $3 \times 6-\mathrm{cm}$ piece of skin for the purpose of flap monitoring. Endto-end anastomosis of the circumflex scapular artery to the lingual artery was performed, and each of the 2 venae comitantes of the circumflex scapular artery was anastomosed to the internal jugular vein in an end-to-side fashion.

No specific postoperative complications occurred, and the monitoring flap was removed 2 weeks after surgery. At the 1-year follow-up, the patient showed an excellent outcome, with both sides of the face being almost symmetric (Fig. 4).
Case 3

A 27-year-old woman presented with a history of 4 STSG procedures at another hospital for the treatment of a third-degree circumferential flame burn on the bilateral lower extremities. On admission, the physical examination revealed circumferential postburn and postoperative scars on the bilateral lower legs, equinus deformity of the right foot, and an immobile right ankle joint due to severe scar contracture. Ischemic contracture of the calf muscles also was suspected, and lengthening of the Achilles tendon was planned, if necessary.

A transverse incision was made on the posterior aspect of the right ankle most affected by postburn scar contracture. Fishmouth incisions were then made at both ends of the incision to adequately release the contracture; however, the patient's right foot was incapable of passive dorsiflexion. Suspecting ischemic contracture of the calf muscles, Z-plasty was performed to sufficiently lengthen the Achilles tendon, and a Steinmann pin was used to fix the right foot at $100^{\circ}$ dorsiflexion. Then, an $11 \times 15$ $\mathrm{cm}$ scapular fascial flap was elevated from the right back to cover the soft tissue defect. The circumflex scapular artery was anastomosed to the posterior tibial artery in an end-to-side fashion, and each of the 2 venae comitantes of the circumflex scapular artery was anastomosed to the lesser saphenous vein and one vena comitans of the posterior tibial artery in an end-to-end fashion.

At 15 months postoperatively, the patient's right foot was capable of active dorsiflexion to $85^{\circ}$ and passive dorsiflexion to $95^{\circ}$. Its aesthetic appearance was acceptable (Fig. 5). 

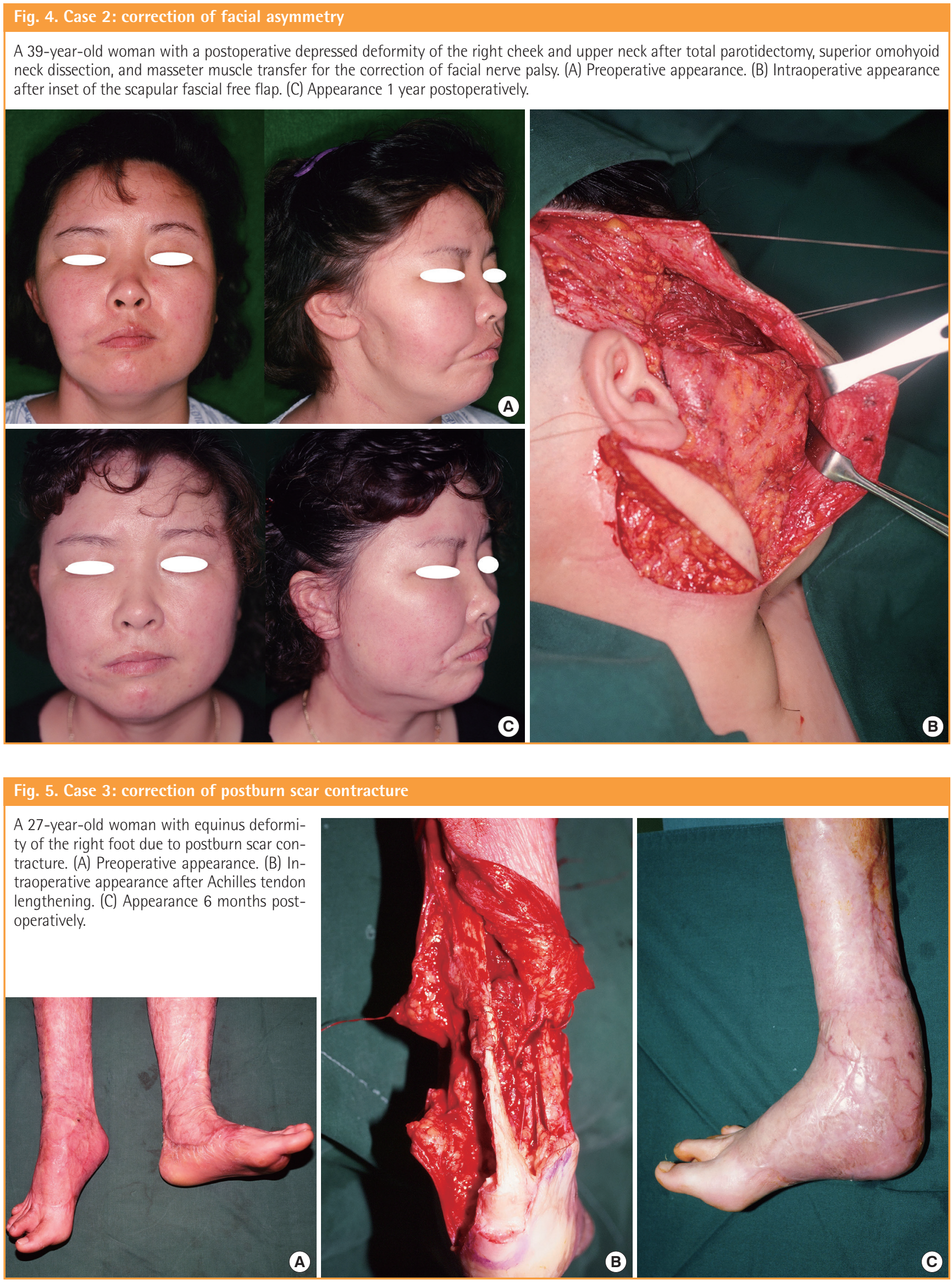


\section{Fig. 6. Case 4: digitation of the scapular fascial flap}
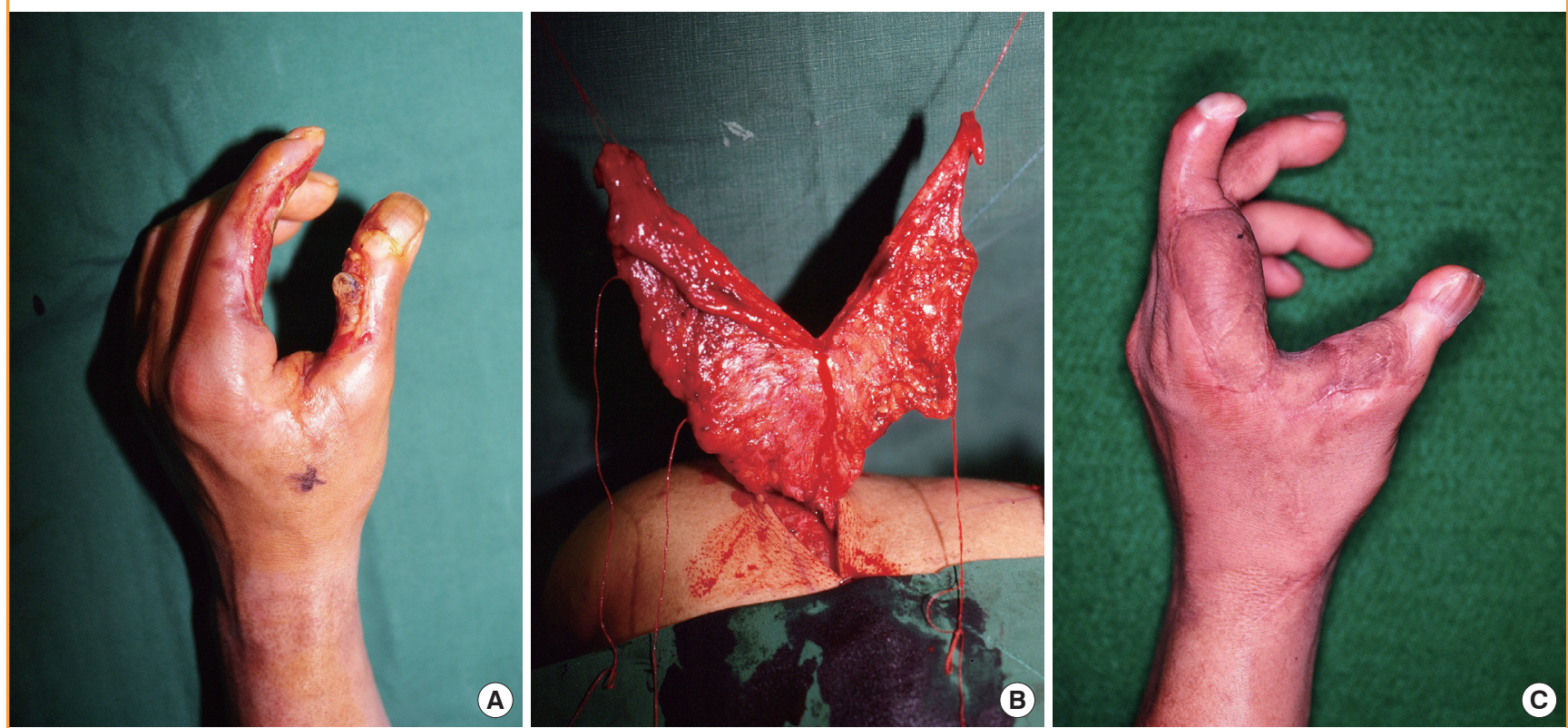

A 42-year-old man with a soft tissue defect of the left thumb and index finger due to an electrical burn. (A) Preoperative appearance. (B) Partial dividing of the scapular fascial flap between the transverse and descending branches of the circumflex scapular artery. (C) Appearance 1 year postoperatively. (D) Donor-site linear scar 1 year postoperatively.

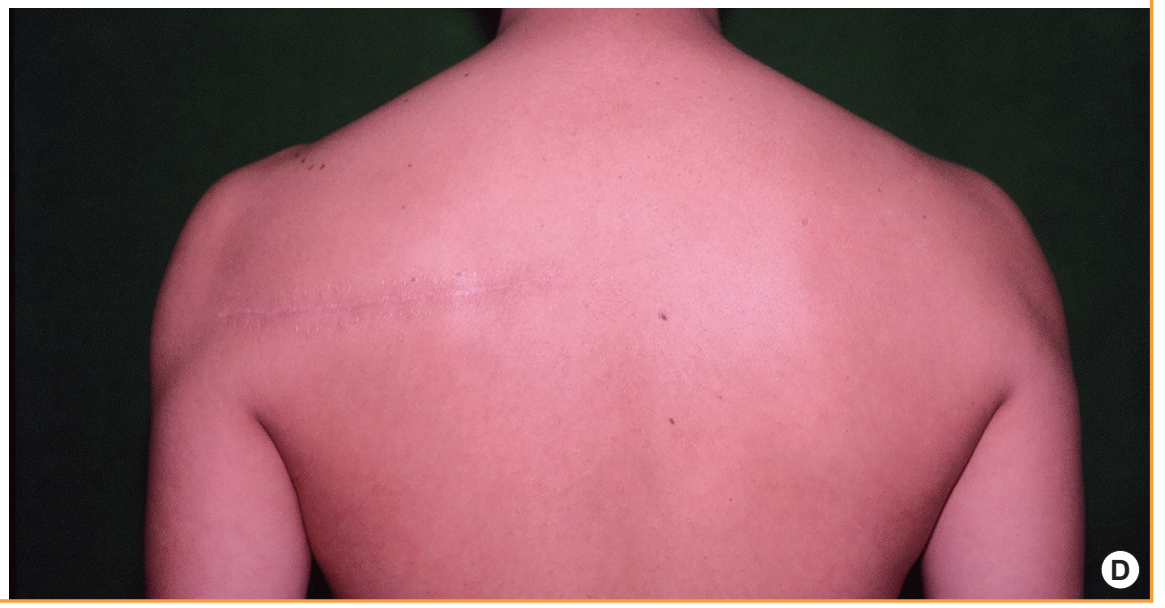

\section{Case 4}

A 42-year-old man presented with a soft tissue defect of the volar surfaces of the left thumb and index finger secondary to a 22,000-volt electrical burn. The flexor pollicis longus tendon of the thumb and the flexor digitorum superficialis tendon of the index finger were partially exposed.

A $9 \times 13-\mathrm{cm}$ scapular fascial flap was harvested and partially divided between the transverse and descending branches of the circumflex scapular artery to cover the raw surface. An STSG was then applied on top of the fascial flap. The circumflex scapular artery was anastomosed to the radial artery in the anatomic snuff box, and each of the 2 venae comitantes of the circumflex scapular artery was anastomosed to the cephalic vein and one vena comitans of the radial artery in an end-to-end fashion.

At the 1-year follow-up, both reconstructed fingers appeared to have natural contours without additional surgery. The donor site had no specific complications other than a linear scar (Fig. 6).

\section{DISCUSSION}

Various methods exist for facial soft tissue augmentation, such as fat grafts, dermal or dermofat grafts, acellular dermal matrix allografts, pedicled flaps, and free flaps. Although fat grafting is relatively easy, in many cases, recorrection is required due to the resorption of some fat within 4 to 12 months. In dermal or dermofat grafting, there are limitations to the volume that can be harvested $[7,8]$. Augmentation using an acellular dermal matrix allograft is relatively simple and does not require a donor site, and augmentation with a large volume can be performed using several layers. However, despite these advantages, some studies have reported that several additional augmentations were required when Cymetra (LifeCell Corporation, Branchburg, NJ, USA) was used due to long-term reductions in volume $[9,10]$. For cases that require a great degree of augmentation, various free flaps have been attempted in accordance with developments 
in microsurgery. These techniques offer 3-dimensional reconstruction of the defect site and yield a better aesthetic outcome than other treatments. Until recently, the free flaps most commonly used for facial soft tissue augmentation have been the omental, groin, latissimus dorsi muscular or musculocutaneous, serratus muscular, scapular fascial, and various perforator flaps [11-15]. Each type of free flap has its advantages and disadvantages. The scapular fascial free flap is exceptionally useful for correcting facial asymmetry and reconstructing the curved regions connecting the eyes, nose, upper lip, and cheeks. In addition, due to its abundant vascular network, circulation is not greatly impaired, even with folding. We have successfully performed soft tissue augmentation by folding the distal third of the scapular fascial flap in patients with a postmaxillectomy or postparotidectomy deformity. Additionally, in cases in which a bony defect of the orbit or mandible is present, simultaneous reconstruction of the bony and soft tissue defects can be performed by harvesting part of the scapular bone with the scapular fascial flap [16].

Similarly, various procedures have been used to cover soft tissue defects of the extremities. Efforts have been made to find a thin flap with sufficient thickness to cover a soft tissue defect of the dorsum of the hand, wrist, distal forearm, foot dorsum, and ankle, where the soft tissue is particularly thin and deep tissue such as bone and tendon is easily exposed.

In 1980, Smith [1] used the temporoparietal fascia to reconstruct soft tissue defects of the lower extremities. Later, in 1987, Kim et al. [2] demonstrated a new fascial flap, known as the dorsal thoracic fascial flap, which used the circumflex scapular vascular system as the pedicle. The dorsal thoracic fascial flap is also known as the scapular fascial flap [3].

The scapular fascia is not only thin and pliable, but also durable. Compared to relatively bulky fasciocutaneous, musculocutaneous, and muscle flaps, the scapular fascia is more suitable for natural contouring, which is an advantage of the adipofascial flap. In addition, it also has a functional advantage in that its surface is adequate for tendon gliding. In 2010, Guimberteau et al. [17] histologically validated the suitability of adipofascial tissue for tendon gliding. In addition, several subsequent studies have reported that adipofascial tissue coupled with tenolysis produces excellent outcomes in patients with continually recurrent tendon adhesion $[18,19]$. The authors have not experienced a case in which additional tenolysis was performed in response to postoperative tendon adhesion. Another benefit of the scapular fascial flap is that it overcomes the limitations of the temporoparietal, radial forearm, and lateral arm fascial flaps. The temporoparietal fascial flap has a short pedicle, is restricted in harvestable size, and the donor site can develop alopecia [20]. The radial forearm fascial flap requires sacrifice of the radial artery, which is one of the main arteries that supply the hand [21]. The lateral arm fascial flap is restricted in harvestable size to $100 \mathrm{~cm}^{2}$, and the donor-site scar is often clearly visible [22].

However, one inconvenience of the scapular fascial flap is that it requires a position change during surgery. Nevertheless, the flap has many benefits. For example, it rarely shows anatomical variation in the vascular pedicle, and more than $350 \mathrm{~cm}^{2}$ can be harvested. The scapular fascial flap can result in the largest size of all fascial flaps [2-6]. Also, as seen in case 4, delicate reconstruction of multiple defects of a specific structure, such as the finger, is possible with digitation of the scapular fascial flap.

Despite these advantages, partial loss of the skin graft applied over the scapular fascial flap during limb reconstruction has been reported in many studies [4-6]. The review carried out by Colen et al. [6] suggested that the abundant vascular network of the fascia may be responsible for increased bleeding from the flap surface, which may interfere with skin graft application. Additionally, according to our clinical experience, as the skin graft is applied to the fascia, part of the fat tissue of the adipofascial flap may be absorbed and cause dehiscence between the graft and the fascia. With regard to skin graft loss, Chen and el-Gammal [23] and many other authors have reported that skin graft engraftment above the fascial flap may be more effective if the skin graft is delayed for 1 week. This delay can provide time for fascial flap hydration, by itself and with wet saline dressing. This wet environment can encourage fibroblasts and endothelial cells to generate more granulation tissue that supports skin graft engraftment. Additionally, Meland and Weimer [24] proposed that the use of a skin substitute, such as a Biobrane (UDL Laboratories, Rockford, IL, USA), to cover the fascia temporarily and to delay the skin graft for 3 to 5 days could minimize such complications. However, Datiashvili and Yueh [5] reported that skin substitutes should be used with caution because they can stick to the fascia, causing the vessels to become vulnerable and resulting in loss of the flap.

In terms of donor-site morbidity, use of the latissimus dorsi muscular or musculocutaneous flap, which is mainly used for the reconstruction of large soft tissue defects of the lower extremity, can lead to measurable reductions in shoulder joint stability, strength, range of motion, and general function in younger patients with a high level of activity. The scapular fascial free flap may be beneficial in this age group because it can cover large defect areas without functional loss at the donor site. Additionally, the scar is typically linear and easily concealed [25]. However, it should be kept in mind that seromas frequently develop at the donor site. Pathophysiologically, acute inflammatory exudate and dead space formed after flap harvesting are the main causes of seromas $[6,26]$. We initially found that seromas fre- 
quently occurred, but minimized their incidence by adjusting certain aspects of the procedure. First, vessel and tissue injury were minimized by using bipolar cautery at a low-energy setting to achieve precise and delicate hemostasis. In addition, simultaneous quilting sutures were performed during donor-site closure to minimize the formation of dead space.

Based on our clinical experience, we have discovered various surgical uses of the scapular fascial free flap. This flap is advantageous because it is thin and pliable, useful for contouring, and durable. It also has a suitable surface for tendon gliding and a reliable pedicle source. Compared with other types of fascial flap, the scapular fascial flap has a larger harvestable size, does not require the sacrifice of muscle at the donor site, and results in minimal scarring. However, when a scapular fascial flap is used in limb reconstruction, an additional skin graft is necessary to cover the flap, and partial loss of the skin graft is common. In these cases, the scapular fascial flap is not as aesthetically effective as a cutaneous flap.

Although the scapular fascial free flap is not widely employed despite its usefulness, it should be considered to be a valuable option for soft tissue coverage of the extremities and facial soft tissue augmentation.

\section{REFERENCES}

1. Smith RA. The free fascial scalp flap. Plast Reconstr Surg 1980;66:204-9.

2. Kim PS, Gottlieb JR, Harris GD, et al. The dorsal thoracic fascia: anatomic significance with clinical applications in reconstructive microsurgery. Plast Reconstr Surg 1987;79:7280.

3. Jin YT, Cao HP, Chang TS. Clinical application of the free scapular fascial flap. Ann Plast Surg 1989;23:170-7.

4. Hazani R, Brooks D, Buntic RF. Resurfacing of a complex upper extremity injury: an excellent indication for the dorsal thoracic fascial flap. Microsurgery 2009;29:128-32.

5. Datiashvili RO, Yueh JH. Management of complicated wounds of the extremities with scapular fascial free flaps. J Reconstr Microsurg 2012;28:521-8.

6. Colen LB, Pessa JE, Potparic Z, et al. Reconstruction of the extremity with the dorsal thoracic fascia free flap. Plast Reconstr Surg 1998;101:738-44.

7. Kanchwala SK, Bucky LP. Facial fat grafting: the search for predictable results. Facial Plast Surg 2003;19:137-46.

8. Mordick TG, 2nd, Larossa D, Whitaker L. Soft-tissue reconstruction of the face: a comparison of dermal-fat grafting and vascularized tissue transfer. Ann Plast Surg 1992;29:390-6.

9. Costantino PD, Govindaraj S, Hiltzik DH, et al. Acellular dermis for facial soft tissue augmentation: preliminary report. Arch Facial Plast Surg 2001;3:38-43.

10. Kridel RW. Acellular human dermis for facial soft tissue augmentation. Facial Plast Surg Clin North Am 2001;9:413-37.

11. Walkinshaw M, Caffee HH, Wolfe SA. Vascularized omentum for facial contour restoration. Ann Plast Surg 1983;10: 292-300.

12. Harashina T, Fujino T. Reconstruction in Romberg's disease with free groin flap. Ann Plast Surg 1981;7:289-94.

13. de la Fuente A, Jimenez A. Latissimus dorsi free flap for restoration of facial contour defects. Ann Plast Surg 1989;22:1-8.

14. Angel MF, Bridges RM, Levine PA, et al. The serratus anterior free tissue transfer for craniofacial reconstruction. J Craniofac Surg 1992;3:207-12.

15. Longaker MT, Siebert JW. Microvascular free-flap correction of severe hemifacial atrophy. Plast Reconstr Surg 1995;96: 800-9.

16. Takeishi M, Ishida K, Makino Y. The thoracodorsal vascular tree-based combined fascial flaps. Microsurgery 2009;29: 95-100.

17. Guimberteau JC, Delage JP, McGrouther DA, et al. The microvacuolar system: how connective tissue sliding works. J Hand Surg Eur Vol 2010;35:614-22.

18. del Pinal F, Moraleda E, de Piero GH, et al. Outcomes of free adipofascial flaps combined with tenolysis in scarred beds. J Hand Surg Am 2014;39:269-79.

19. Ozakpinar HR, Tellioglu AT, Eryilmaz T, et al. A reliable option for wrist soft tissue defects: adipofascial flaps for immediate and late reconstruction. Int Wound J 2013;10:661-5.

20. Woods JM 4th, Shack RB, Hagan KF. Free temporoparietal fascia flap in reconstruction of the lower extremity. Ann Plast Surg 1995;34:501-6.

21. Jones NF, Jarrahy R, Kaufman MR. Pedicled and free radial forearm flaps for reconstruction of the elbow, wrist, and hand. Plast Reconstr Surg 2008;121:887-98.

22. Lai CS, Tsai CC, Liao KB, et al. The reverse lateral arm adipofascial flap for elbow coverage. Ann Plast Surg 1997;39: 196-200.

23. Chen HC, el-Gammal TA. The lateral arm fascial free flap for resurfacing of the hand and fingers. Plast Reconstr Surg 1997;99:454-9.

24. Meland NB, Weimar R. Microsurgical reconstruction: experience with free fascia flaps. Ann Plast Surg 1991;27:1-8.

25. Smith SL. Functional morbidity following latissimus dorsi flap breast reconstruction. J Adv Pract Oncol 2014;5:181-7.

26. Agrawal A, Ayantunde AA, Cheung KL. Concepts of seroma formation and prevention in breast cancer surgery. ANZ J Surg 2006;76:1088-95. 